\title{
Naturaleza de la ciencia
}

\section{en un objeto virtual de aprendizaje para el profesorado de ciencias en formación}

\section{Nature of science in a virtual learning object for prospective science teachers}

Filipe Faria Berçot

Instituto de Biociencias, Universidad

de San Pablo, San Pablo, Brasil.

bercot@ib.usp.br

\author{
Andrea Revel Chion \\ Instituto CeFIEC, Universidad de Buenos \\ Aires, Buenos Aires, Argentina. \\ andrearevelchion@gmail.com
}

\author{
Agustín Adúriz-Bravo \\ CONICET/Instituto CeFIEC, Universidad \\ de Buenos Aires, Buenos Aires. Argentina. \\ aadurizbravo@cefiec.fcen.uba.ar
}

RESUMEN • Nos propusimos validar un objeto virtual de aprendizaje (OVA) sobre un episodio de historia de la ciencia del siglo XVIII para enseñar naturaleza de la ciencia (NOS). La aplicación del OVA en formación de profesorado permitió su evaluación en contexto. El episodio histórico elegido concernió los estudios del naturalista Charles Bonnet, que construyó argumentos en favor de la idea de reproducción asexual en animales. Desarrollamos el OVA como sitio web, guiados por presupuestos teóricos sobre el uso de narrativas en la educación científica; elaboramos una "historia de la ciencia» con textos breves y material de fuentes primarias. En la narrativa intercalamos indagaciones basadas en problemas auténticos para abordar la NOS. El análisis de la aplicación del OVA con futuros profesores de biología evidenció resultados positivos en términos de la identificación de algunas «ideas clave» de NOS.

PALABRAS CLAVE: Objeto virtual de aprendizaje; Historia de la ciencia; Naturaleza de la ciencia; Formación del profesorado de ciencias.

ABSTRACT - Our aim was to validate a virtual learning object (VLO) on an episode of the history of science in the $18^{\text {th }}$ century to teach the nature of science (NOS). Implementation of the VLO in pre-service teacher education permitted to assess it in context. The historical episode that we selected concerned the studies of the naturalist Charles Bonnet, who produced arguments in favour of the idea of asexual reproduction in animals. We developed our VLO as a website, based on theoretical guidelines on the use of narratives in science education; we wrote a «science story» with short texts and material from primary sources. The narrative was interspersed with little pieces of enquiry based on authentic problems to discuss NOS. Analysis on the implementation of the VLO with prospective biology teachers produced positive results in terms of the identification of some «key ideas» of NOS.

KEYWORDS: Virtual learning object; History of science; Nature of science; Science teacher education.

Recepción: agosto 2019• Aceptación: mayo 2020 • Publicación: marzo 2021

Faria Berçot, F., Revel Chion, A. y Adúriz-Bravo, A. (2021).Naturaleza de la ciencia en un objeto virtual de aprendizaje para el profesorado de ciencias en formación. Enseñanza de 


\section{INTRODUCCIÓN}

La investigación aquí reportada tuvo como objetivo general validar, con profesorado de ciencias experimentales en formación, la utilidad didáctica de un objeto virtual de aprendizaje construido sobre un episodio de historia de la biología del siglo XviII con el fin de poner en valor algunos aspectos de la naturaleza de la ciencia.

Desde hace tres décadas la investigación en didáctica de las ciencias busca comprender las concepciones sobre la ciencia que sostienen estudiantado y profesorado; tal preocupación constituye la línea de trabajo conocida como "naturaleza de la ciencia» (NOS en inglés). Sus resultados de investigación empírica muestran que, en todos los niveles indagados, las personas muestran concepciones «deformadas» del quehacer científico (Abell y Smith, 1994; Fernández et al., 2002).

De acuerdo con una gran nómina de autores (Erduran y Wong, 2013; García-Martínez e Izquierdo-Aymerich, 2014), esta problemática se debe en parte a los documentos curriculares y materiales educativos y en parte a las prescripciones de enseñanza y de evaluación, todo excesivamente enfocado en los contenidos conceptuales de la ciencia escolar. La casi exclusiva preocupación por la estructura de las disciplinas que hay que enseñar, presentada en su «forma final», en desmedro de los aspectos epistémicos (fundamentales para la alfabetización científica) aparece también en la formación del profesorado, lo que instala un modelo que logra poca comprensión significativa de la ciencia en los estudiantes (Mulvey y Bell, 2016).

Es en este contexto en el que el presente trabajo se centra en el diseño, puesta en marcha y evaluación de un objeto virtual de aprendizaje (OVA) que permita discutir con concepciones «informadas» (fundamentadas en la filosofía de la ciencia reciente) sobre aspectos de la NOS. Nuestra propuesta pretende proveer a los docentes herramientas para que ellos ayuden a sus estudiantes a entender cómo se lleva adelante la ciencia, a apropiarse de los conocimientos por ella producidos y a tomar decisiones fundamentadas sobre cuestiones científicas (Kampourakis, 2016). El OVA que diseñamos, titulado «El enigma del pulgón», recoge un episodio histórico de la segunda mitad del siglo XviII en el que se leyeron evidencias y se construyeron argumentos para conceptualizar una nueva forma de reproducción animal hasta entonces desconocida.

Filosofía e historia de la ciencia han sido muy utilizadas para contribuir a la mejora de la enseñanza de y sobre las ciencias en la formación docente (Adúriz-Bravo, 2011; Rudge et al., 2012). Los investigadores del campo de las contribuciones de las metaciencias a la educación científica (Adúriz-Bravo, 2011, 2015b; Allchin, 2013; Matthews, 2012) destacan algunos beneficios que proporciona la historia de la ciencia: facilitar la comprensión de conceptos y procedimientos de la ciencia; mostrar que los científicos crean hipótesis partiendo de las ideas con que cuentan y mediante analogías; ayudar a identificar que ellos poseen preconcepciones que moldean observaciones y experimentos; reconocer el carácter provisorio y situado de las teorías científicas. Una enseñanza de las ciencias de calidad y para todos pretende, a partir de esos y otros aspectos epistemológicos y con los contextos provistos por la historia de la ciencia, mostrar al estudiantado que el desarrollo del conocimiento científico es arduo y complejo y no sigue fórmulas metodológicas infalibles.

Esta nueva enseńanza de las ciencias que se apoya fuertemente en la naturaleza de la ciencia echa mano muchas veces de lo que podemos llamar «historias de la ciencia», que son narrativas de base histórica. Tales historias, como la que aquí presentamos, pueden utilizarse en diversas modalidades y formatos. Nos decantamos por una estrategia virtual, en primer lugar, porque no hay muchas propuestas virtuales que utilicen episodios de historia de la ciencia para enseñar naturaleza de la ciencia desde la perspectiva de la indagación y, en segundo lugar, porque los ambientes virtuales son medios de amplia difusión y alcance. 
En este trabajo optamos por desarrollar un producto que integre la historia de la ciencia y la tecnología informática dentro de la categoría amplia de materiales educativos que se llaman «objetos virtuales de aprendizaje»; son ejemplos de OVA contenido instruccional multimedia, software educativo, sistemas expertos y tantos otros afines (Wiley, 2002). En este contexto, nuestro OVA buscó integrar, en un texto bien delimitado, una narrativa histórica dirigida a crear un ambiente de aprendizaje basado en la indagación. Dentro de la narrativa del OVA insertamos problemas históricos auténticos con el fin de contribuir, mediante la indagación, al aprendizaje de aspectos de naturaleza de la ciencia en profesores de ciencias en formación.

\section{EL EPISODIO HISTÓRICO}

El episodio histórico utilizado presenta el «descubrimiento» de la multiplicación sin apareamiento (partenogénesis) en pulgones, realizado por el naturalista Charles Bonnet (1720-1793) a mediados del siglo XVIII en Ginebra. Por aquella época el fenómeno de la generación de los seres vivos era central en el campo de la historia natural. La aparición de evidencias empíricas plausibles de una forma de reproducción animal antes inconcebible causó reajustes conceptuales y la consolidación de nuevos modelos metodológicos de investigación. La historia de la ciencia que utilizamos en nuestro OVA retrata también las relaciones de Bonnet con el francés René A. F. de Réaumur (1683-1757), renombrado científico de la época, entre cuyas contribuciones destaca el estudio de los «insectos», término empleado en la época para referirse a «toda clase de organismos pequeños» (Prestes, 2008, p. 270).

La narrativa que construimos se apoyó en un previo estudio histórico riguroso (siguiendo la metodología de investigación propia de la historia de la ciencia), que se realizó a través del análisis de fuentes primarias de Bonnet y Réaumur ${ }^{1}$ y fuentes secundarias de historiadores de la ciencia que investigaron las contribuciones de ambos naturalistas (Berçot y Prestes, 2017).

\section{La «insectología» de Réaumur y Bonnet}

Charles Bonnet, de familia aristocrática ginebrina, obtuvo un título en Leyes a los 23 años; sin embargo, nunca desempeñó la profesión de abogado. Desde la adolescencia su vocación se inclinaba hacia la historia natural de los insectos. Fue un asiduo lector de los trabajos de Réaumur y sabía que él era un activo divulgador de estudios científicos y reconocía a los colaboradores que le enviaban organismos y relatos de observaciones (Terrall, 2010).

Siguiendo el espíritu enciclopedista de la época, Réaumur sabía que no conseguiría elucidar él solo el estudio de los insectos. La «insectología» dependía de los recursos materiales, pero también de la contribución intelectual de personas de diferentes lugares. Él anhelaba expandir el conocimiento acerca de la morfología, comportamiento y aspectos útiles y nocivos de los insectos (Terrall, 2010) y se interesaba por todo aquello que concernía a una singular especie: los pulgones. ${ }^{2}$

\section{El enigma del pulgón}

Entre los naturalistas dedicados al estudio de la perpetuación animal a inicios del siglo Xvin había consenso en torno al hecho de que el proceso de reproducción en animales involucraba ambos sexos.

1. Bonnet (1745) y Réaumur (1737-1742).

2. Los áfidos, popularmente conocidos como pulgones, son insectos del orden Hemíptera, que viven de la savia de las plantas. Actualmente se reconocen unas 4700 especies entre tres familias principales. 
Sin embargo, desde el siglo XviI eruditos y estudiosos aficionados que observaron diferentes especies de pulgones no fueron capaces de identificar ningún tipo de cópula entre esos insectos, hecho que intrigó y motivó investigaciones sobre su reproducción.

Para ir más allá en las observaciones reportadas, Réaumur elaboró un procedimiento nuevo para la época, que denominó «secuestro» (Prestes, 2003). El método consistía en la observación de un organismo aislado de otros similares. Luego de aislar un individuo, Réaumur esperó a que en soledad diera a luz a una cría e inmediatamente encerró a esta, lo que le garantizaba que el recién nacido estuviera libre de contacto con otros insectos. A pesar del celo puesto en el experimento, la cría no alcanzó la edad reproductiva. Los fracasos llevaron a Réaumur a abandonar la cuestión, pero, como era su costumbre, propuso el desafío a sus colegas: «He intentado esta experiencia varias veces, pero ella no ha tenido éxito. Pero relato lo que hice para que otros puedan intentarlo» (Réaumur, 1737, p. 329).

Decidido a rehacer el estudio de Réaumur, Bonnet inició la experiencia con los pulgones en mayo de 1740. Después de haber ideado un medio seguro de mantener el organismo aislado, así describió e ilustró el procedimiento adoptado (el texto se acompańa de su figura original: figura 1):

En un florero, lleno de tierra común, enterré, hasta su cuello, una botella de vidrio llena de agua. Inserté en esa botella una pequeña rama de evónimo, conteniendo no más de cinco o seis hojas, después de examinarlas por todos los lados con la mayor atención. A continuación, deposité sobre una de esas hojas un pulgón cuya madre, desprovista de alas, acababa de dar a luz bajo mis ojos. Cubrí la pequeńa rama con un bote de cristal cuyos bordes encajaban perfectamente sobre la superficie de la tierra del florero. (Bonnet, 1745, p. 27).

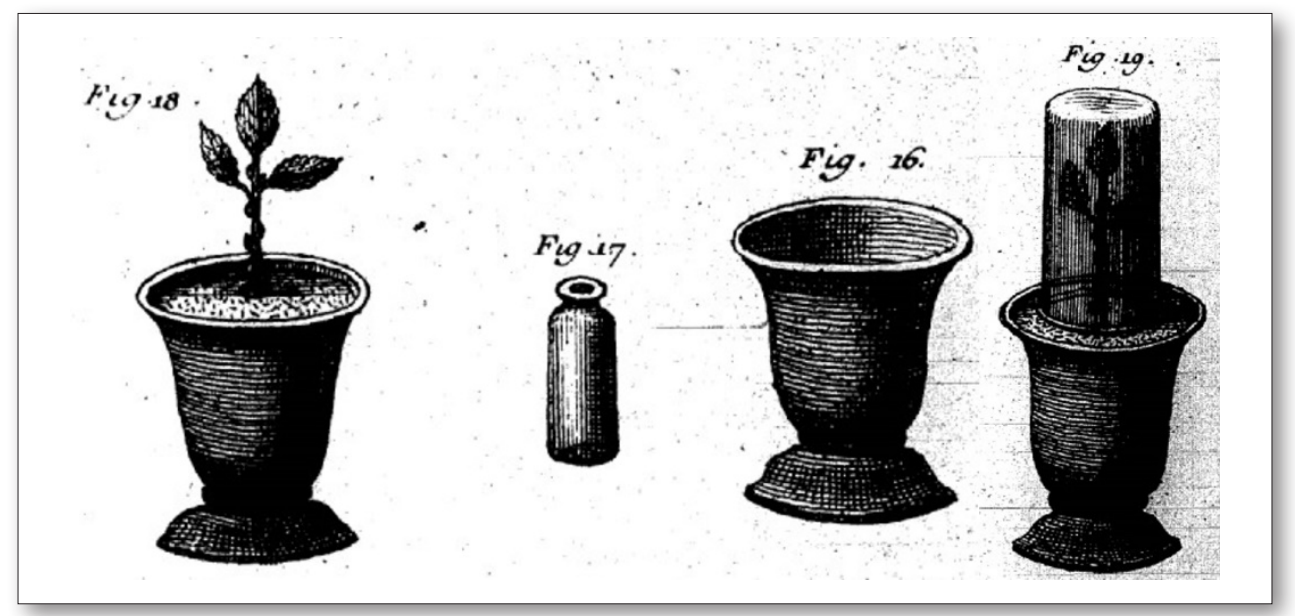

Fig. 1. Ilustración del procedimiento de Bonnet para mantener el pulgón aislado. Fuente: Bonnet (1745, p. 293).

El primer mérito de Bonnet fue haber tenido éxito en la adaptación del dispositivo de aislamiento propuesto por Réaumur, siendo capaz de superar dificultades técnicas que había encontrado el naturalista francés (Sigrist, 2001). También realizó continuos períodos de observación del pulgón y produjo un registro diario, lo que denota su preocupación con los valores epistémicos clásicos que Allchin (2013) denomina «exactitud» y "precisión», aspectos centrales de la dimensión metodológica del quehacer científico.

Después de haber garantizado la eficiencia del mecanismo de aislamiento y supervivencia del insecto, Bonnet pasó a investigar si los pulgones serían, de hecho, «suficientes a sí mismos» (Bonnet, 1745, p. 116). Tras numerosas observaciones, en junio de 1740 su dedicación fue recompensada: 
pudo reportar el nacimiento de 95 crías a lo largo de 21 días (Bonnet, 1745, p. 39). Después de la comunicación de su «descubrimiento», Réaumur divulgó lo reportado por Bonnet a otros tres «excelentes observadores», solicitándoles que repasaran la experiencia (Réamur, 1742, p. 538). Todos ellos obtuvieron la misma conclusión: los pulgones se generaban sin cópula. Para fortalecer su argumento, Bonnet condujo otras «series de experiencias», que repetía y variaba a fin de garantizar la estabilidad y analizar la extensión del «nuevo hecho» (Sigrist, 1994). Tales «series de experiencias», características de los experimentadores del siglo XVIII, remiten a la planificación de experimentos enlazados con el objetivo de corroborar una hipótesis y aportar a la validación de una teoría (Prestes, 2003).

Consideramos que el episodio histórico que elegimos, que en el relato historiográfico tradicional se considera el «descubrimiento» de la multiplicación sin apareamiento, constituye un vehículo potente para la enseñanza de aspectos específicos de la naturaleza de la ciencia.

\section{METODOLOGÍA}

\section{Desarrollo e implementación del OVA}

La elaboración del OVA fue conducida según el objetivo de adecuar la historia de la ciencia con fines didácticos; la narrativa histórica incluida funciona como «telón de fondo» que da sentido al contenido de naturaleza de la ciencia que se quiere enseñar (Adúriz-Bravo, 2009, 2011, 2015b). Aplicamos algunas de las directrices usuales que la literatura recomienda para la construcción de una narrativa didáctica, tales como que tuviera un formato de prosa simple y no técnica y que no excediera el límite de las diez páginas de texto escrito.

De la profusa teorización sobre narrativas (Norris et al., 2005) contemplamos varios aspectos: la presencia de un agente «narrador» que determina la trama histórica y selecciona la secuencia de eventos; el uso del tiempo pasado; la estructura con inicio, desequilibrios y final; el desenlace constituido por la presentación de un éxito o un fracaso. Consideramos la noción teórica de «agencia»: nuestra narrativa involucra a varios seres humanos en el papel de actores que provocan y experimentan eventos estructurados en «momentos».

Con el objetivo de motivar al profesorado destinatario de la narrativa, incluimos elementos contextuales extraídos de la historia de la ciencia; en la dirección de que ellos pudieran aprender cuestiones sobre el quehacer científico introdujimos discusiones relativas a la naturaleza de los procesos de investigación. Este uso de la historia de la ciencia para aprender sobre la naturaleza de la ciencia ha sido ampliamente explotado y difundido desde hace tiempo por educadores e investigadores en didáctica de las ciencias (Adúriz-Bravo, 2011, 2015b; Allchin, 2013; Fouad et al., 2015).

Para estructurar nuestro OVA con la estrategia de indagación elegimos la modalidad de «narrativa interrumpida" (Roach y Wandersee, 1995): el texto principal de la historia de la ciencia aparece en fragmentos autocontenidos, entre los cuales entremezclamos preguntas de indagación que remiten a la reflexión explícita sobre NOS. Las «interrupciones» son pequeñas actividades que hemos denominado «momentos para pensar»; persiguen incentivar en los aspirantes a profesores el razonamiento y la discusión y están estructuradas en torno a cuestiones auténticas extraídas de la propia historia de la ciencia (figura 2). 


\section{\#4 iMomento para pensar!}

Si todos los animales conocidos, desde los elefantes hasta las moscas más pequeñas, requieren apareamiento para su perpetuación, ¿los áfidos serían una excepción a esta regla? ¿Cómo habrían explicado este fenómeno los naturalistas de la época?

Ingresen aquí su respuesta...

Fig. 2. Ejemplo de cuestión de indagación sobre NOS insertada en el OVA.

Las cuestiones insertadas proporcionan oportunidades para explorar aspectos de la naturaleza de la ciencia. Al intentar responderlas, los profesores destinatarios del OVA son invitados a ponerse en el lugar de los científicos del pasado y tratar de encontrar posibles soluciones en un contexto histórico original. Se ponen así a consideración problemas epistémicos y metodológicos que apuntan a comprender, por ejemplo, el papel de los sesgos sociales en la construcción de la ciencia o los desafíos relativos a la comunicación de las ideas a los colegas y al gran público (Allchin, 2013).

La aplicación del OVA, durante el segundo semestre de 2017, se realizó en una institución pública de formación docente inicial de la ciudad de Buenos Aires, Argentina, con 29 estudiantes de la carrera de grado de Profesorado en Biología que dieron su consentimiento para formar parte de este estudio. El primer autor de este trabajo fue invitado a ser docente de una clase de dos horas de reloj en una asignatura de didáctica de la biología. Los estudiantes se organizaron en 10 grupos de entre dos y tres miembros cada uno y resolvieron en grupos las tareas propuestas en el OVA, consignando sus respuestas en los campos interactivos dispuestos a tal propósito (como el que se ve en la figura 2). Al final de la clase respondieron, de forma individual, un cuestionario escrito abierto destinado a valorar la aplicabilidad del OVA en la educación secundaria y luego discutieron en plenario con el profesor-investigador sus percepciones sobre la calidad del recurso y sus posibles objetivos y expectativas de aprendizaje.

Las respuestas electrónicas grupales subidas a los campos interactivos del OVA y las respuestas manuscritas individuales al cuestionario de aplicabilidad constituyen los insumos empíricos que analizamos aquí.

\section{Metodología de análisis de datos}

Para diagnosticar las concepciones de los participantes de este estudio sobre distintos aspectos vinculados a la naturaleza de la ciencia se optó por la utilización de rúbricas, entendidas como instrumento de evaluación crítica que determina un conjunto de criterios que analizar y, para cada uno de ellos, niveles de gradación o de calidad (Hafner y Hafner, 2003; Reddy y Andrade, 2010). Las rúbricas, en cuanto que son instrumentos provenientes del campo educativo (evaluación de los aprendizajes), permiten eventualmente comunicar a los sujetos bajo estudio «expectativas de logro» y proporcionarles retroalimentación para la mejora (Andrade et al., 2008). Por el mismo motivo poseen mayor robustez a la hora de analizar los desempeños referentes a habilidades de conocimiento y de práctica científicas (biología) y metacientíficas (naturaleza de la ciencia) (Acevedo-Díaz et al., 2017; Berçot et al., 2018). 
El desarrollo de las rúbricas siguió algunos de los criterios clásicos establecidos por la literatura (Moskal y Leydens, 2000; Reddy y Andrade, 2010); se describe en el cuadro 1. Se construyó una rúbrica para cada uno de los siete «momentos para pensar» que incluía el OVA.

\section{Cuadro 1.}

Elementos estructurales de toda rúbrica

\begin{tabular}{|l|l|}
\hline Criterios de evaluación & $\begin{array}{l}\text { Indicadores predefinidos: son elementos que permiten la conceptualización y definición de pa- } \\
\text { trones. En el caso del OVA usado en este estudio, los criterios remiten a aspectos vinculados a } \\
\text { la naturaleza de la ciencia. }\end{array}$ \\
\hline Definiciones de calidad & $\begin{array}{l}\text { Descripción detallada de lo que se espera de un estudiante para mostrar habilidad y alcanzar un } \\
\text { nivel específico. El «grado de dominio» para cada criterio de evaluación discierne capacidades } \\
\text { de argumentar. }\end{array}$ \\
\hline $\begin{array}{l}\text { Estrategias de puntua- } \\
\text { ción (scoring) }\end{array}$ & $\begin{array}{l}\text { Escala para interpretar o clasificar la comprensión del proceso en los sujetos evaluados. Se basa } \\
\text { en listas de contenidos, habilidades o explicaciones «tipo» que orientan la categorización de las } \\
\text { respuestas. }\end{array}$ \\
\hline
\end{tabular}

Cada rúbrica atiende a un aspecto epistemológico particular de la naturaleza de la ciencia e identifica sucesivos niveles de sofisticación del discurso explicativo del profesorado sobre ese aspecto. Por ejemplo, en la cuestión que apuntaba a abordar la llamada "carga teórica en la observación», para hablar de una comprensión aceptable consideramos necesario tener conciencia del papel del background teórico de los científicos en la interpretación de datos, la relevancia de sus concepciones previas en la lectura del mundo y la influencia de las experiencias personales y el contexto sociocultural en la construcción de ideas.

Para los fines de este estudio optamos por la utilización de «rúbricas holísticas», que proporcionen descripciones más generales y sin categorizaciones excesivas, pero que al mismo tiempo sean suficientes para comprender lo que se espera de quienes responden y brinden un análisis bien definido de lo que se solicitó (Allen y Tanner, 2006). El criterio de gradación en tres niveles es pertinente, pues permite analizar las respuestas de forma general y a la vez rápida y consistente.

Las rúbricas pasaron por un proceso de validación con profesores y estudiantes de postgrado en didáctica y filosofía de la ciencia para adecuarse a las preguntas del OVA y a los aspectos de NOS bajo estudio, ajustar la calidad de la redacción y refinar los niveles de gradación. Este último punto merece ser destacado, pues el nivel 2 (intermedio) fue el que ofreció más dificultad para su definición: demandó discusiones hasta obtener un grado de consenso aceptable.

Desde un abordaje metodológico cualitativo, los datos de este trabajo fueron tratados a la luz de la técnica (categorial) del análisis de contenido (Bardin, 1977). Una de las etapas de esta técnica se refiere a la exploración y lectura «flotante» del material recogido (respuestas a las cuestiones de naturaleza de la ciencia). En el proceso se fueron seleccionando las palabras y expresiones que se relacionaban directamente con cada criterio y grado de las rúbricas, de modo que fuera posible reducir las respuestas integrales de los respondientes a categorías más sencillas, tipificadas.

\section{RESULTADOS Y DISCUSIÓN}

\section{Aspectos de NOS trabajados en el OVA}

El primer aspecto NOS discutido en el OVA remite al papel de la "carga teórica» en las observaciones. Sobre la base del contexto histórico se elaboró, en el «Momento para pensar 2», una cuestión en torno a por qué diversos naturalistas propusieron explicaciones muy distintas para un mismo fenómeno. El cuadro 2 representa los tres niveles (indicadores) de respuesta esperados para esa pregunta, organizados en grado de robustez decreciente. 
Cuadro 2.

Rúbrica sobre la carga teórica de la observación

¿Por qué estos naturalistas propusieron explicaciones tan distintas a pesar de que habian observado el mismo fenómeno?

\begin{tabular}{|l|l|}
\hline Nivel 3 & $\begin{array}{l}\text { Se espera que el estudiante explique que los naturalistas de la época, así como los científicos actuales, poseen } \\
\text { conocimientos previos y creencias personales de carácter social, cultural, político, religioso, etc., que son orien- } \\
\text { tados por las teorías de las cuales son defensores, que tienen diferentes patrones de razonamiento y que la } \\
\text { observación y la interpretación de los fenómenos se cargan de juicios particulares. }\end{array}$ \\
\hline Nivel 2 & $\begin{array}{l}\text { Se espera que el estudiante argumente que las explicaciones de los científicos dependen de sus conocimientos } \\
\text { previos y de las teorías vigentes, que el contexto en que el observador está inserto influye en la interpretación } \\
\text { y que las creencias personales pueden afectar la observación. }\end{array}$ \\
\hline Nivel 1 & $\begin{array}{l}\text { Se espera que el estudiante argumente que explicaciones distintas proceden de la falta de interpretación o de } \\
\text { interpretaciones erróneas de las evidencias; que los científicos siguen un método objetivo y que, con más datos } \\
\text { y más tecnología, serán capaces de llegar a consensos o explicaciones mejores. }\end{array}$ \\
\hline
\end{tabular}

Cuadro 3.

Categorías elaboradas para evaluar el aspecto NOS: «carga teórica en la observación»

\begin{tabular}{|l|l|c|}
\hline \multicolumn{1}{|c|}{ Grupos } & \multicolumn{1}{|c|}{ Categorías creadas } & Nivel \\
\hline $1,2,4,5,7$ y 10 & Debido a creencias personales o conocimientos previos & 2 \\
\hline $3,6,8$ y 9 & Debido a métodos distintos de observación & 1 \\
\hline
\end{tabular}

En el cuadro 3 puede verse que, para la mayoría de los participantes en este estudio, el origen de la variación en las explicaciones radica en los puntos de vista del observador que, a su tiempo, se basan en conocimientos previos y creencias personales; por esta razón, clasificamos a esos grupos de profesores en formación en el nivel 2. Con el propósito de justificar este análisis reproducimos algunos extractos de las respuestas de los grupos. El siguiente es un ejemplo de respuesta considerada como de nivel 2:

Los tres naturalistas llegaron a distintas hipótesis ya que cada uno tiene concepciones previas distintas, y lo que observaron lo relacionan con ellas enfocándose en diferentes detalles observados (grupo 10).

Ejemplo de respuesta considerada como de nivel 1:

Porque los tres usaron métodos de observación distintos, generando tres hipótesis diferentes, la hipótesis de Cestoni fue la más acertada mostrando que él tenía mayor conocimiento teórico (grupo 3).

Nuestros hallazgos coinciden con lo reportado en la literatura. Por ejemplo, el trabajo de Lozano y colaboradores (2016) discute un episodio histórico referente a la construcción de un modelo de membrana celular en el siglo XIX con el propósito de abordar el aspecto relativo a la carga teórica en los científicos. Según los autores del estudio, debatir con profesores en formación la cuestión referente a las observaciones cargadas de teoría permite desafiar el preconcepto tan establecido del «descubrimiento» como la actividad central de la investigación científica. La discusión comparativa entre distintas respuestas a esta cuestión supone considerar un modelo epistemológico que actualmente se juzga más apropiado: la idea de que los hechos científicos se "construyen» en una interacción laboriosa entre mundo e ideas (Lozano et al., 2016, p. 54).

Otro aspecto de NOS discutido en el OVA fue el papel de las explicaciones alternativas aportadas por los científicos ante resultados no esperados. Esto es, ¿qué significa afirmar que se ha identificado un «mal resultado» frente a una pregunta de investigación y cómo se puede «salvar» ese resultado? El cuadro 4 presenta el «momento para pensar 5» con su consigna y sus niveles. 
Cuadro 4.

Rúbrica sobre las explicaciones alternativas aportadas por los científicos

\begin{tabular}{|l|l|}
\hline $\begin{array}{l}\text { Conociendo la experiencia de Réaumur y su minuciosidad experimental y metodológica, ¿cuáles son las razones por las que su } \\
\text { experimento no logró los resultados esperados? ¿Qué significa este «mal resultado» en relación con la pregunta de investigación? }\end{array}$ \\
\hline Nivel 3 & $\begin{array}{l}\text { Se espera que el estudiante comprenda que un resultado no esperado no implica que el fenómeno bajo } \\
\text { estudio no ocurra, que los científicos buscan proponer interpretaciones distintas a partir de los resultados } \\
\text { observados, que dificultades metodológicas como el control de variables y la cantidad de repeticiones experi- } \\
\text { mentales pueden impactar en los resultados obtenidos. Se espera, asimismo, que el estudiante sea consciente } \\
\text { de que observaciones y experimentos solo ayudan a aportar evidencias para las preguntas planteadas y no } \\
\text { constituyen «pruebas» demostrativas. }\end{array}$ \\
\hline Nivel 2 & $\begin{array}{l}\text { Se espera que el estudiante mencione que un resultado no esperado puede ser fruto de las dificultades me- } \\
\text { todológicas y que entienda que los datos empíricos solo proporcionan evidencias para soportar hipótesis. }\end{array}$ \\
\hline Nivel 1 & $\begin{array}{l}\text { Se espera que el estudiante adhiera a la idea de que el mal resultado significa que la pregunta de investigación } \\
\text { es errónea, que un fenómeno no ocurre en el mundo natural cuando no puede ser probado por un experi- } \\
\text { mento. }\end{array}$ \\
\hline
\end{tabular}

\section{Cuadro 5.}

Categorías elaboradas sobre el papel de las explicaciones alternativas aportadas por los científicos

\begin{tabular}{|l|l|c|}
\hline \multicolumn{1}{|c|}{ Grupos } & \multicolumn{1}{|c|}{ Categorías creadas } & Nivel \\
\hline $2,4,6$ y 10 & Debido a problemas experimentales & 2 \\
\hline $1,3,5,7,8$ y 9 & Porque la hipótesis podría estar errada & 1 \\
\hline
\end{tabular}

Con respecto a este aspecto, cuatro grupos de profesores en formación presentaron concepciones parcialmente adecuadas, al alegar que los resultados no deseados podrían ser fruto de problemas y dificultades experimentales (cuadro 5). Sin embargo, la mayoría de los sujetos, al suponer que la causa de que los datos observados fueran distintos de los esperados es la elección equivocada de hipótesis, muestran una concepción poco informada acerca de este aspecto de la naturaleza de la ciencia, que se acerca a la visión empírico-inductivista, en la cual los datos "hablan por sí solos», con independencia de la interpretación del observador.

Las posiciones de los grupos de estudiantes se pueden observar en las siguientes respuestas textuales. Ejemplo de respuesta considerada como de nivel 1:

El experimento no logró los «resultados esperados» porque había un solo individuo, al no haber cópula no se reprodujo. El mal resultado se refiere a que se hizo la experimentación bajo la hipótesis de que se autofecunda (grupo 3).

Ejemplo de respuesta considerada como de nivel 2:

Faltaba manejar mayor número de variables (por ejemplo, otras pruebas en diferentes condiciones) (grupo 6).

Se debe considerar, sin embargo, que ningún grupo mencionó el hecho de que resultados inesperados a veces necesitan explicaciones teóricas alternativas (nivel 3). En ciertas circunstancias, resultados inesperados no son sinónimo de yerros experimentales o falta de rigor metodológico, sino de contradicción o confrontación con el cuerpo teórico vigente. En ese sentido, corresponde al científico reconocer esas rupturas teóricas y avanzar hacia la postulación de explicaciones alternativas que «cubran» las nuevas observaciones. 
En la discusión de esta idea epistemológica sofisticada se movilizan conocimientos metateóricos relacionados con la cuestión del apoyo con que cuentan las explicaciones científicas rigurosas (¿cómo hemos hecho para saber esto? o ¿qué indicadores y evidencias son necesarios para estar seguros?) (Abell y Smith, 1994; Allchin, 2013).

El OVA también nos permitió, a través del «momento para pensar 6», investigar las concepciones de los profesores participantes acerca de las prácticas y métodos empíricos empleados en el desarrollo del conocimiento científico (cuadro 6).

\section{Cuadro 6.}

Rúbrica sobre el papel de las prácticas y los métodos experimentales

\begin{tabular}{|c|c|}
\hline Nivel 3 & $\begin{array}{l}\text { Se espera que el estudiante argumente que las evidencias obtenidas por Bonnet fueron construidas desde } \\
\text { modos rigurosos de intervención sobre la naturaleza. También que tales modos se basan en la interpretación } \\
\text { propia y cargada de teoría de sus observaciones e inferencias. A su tiempo, que los métodos empíricos con- } \\
\text { fiables dependen del control de variables, la replicación, el tamaño de la muestra y muchos otros recaudos. Se } \\
\text { espera que enfaticen la importancia de la formulación de hipótesis aceptables sobre el fenómeno investigado } \\
\text { a fin de fortalecer o apoyar una afirmación. }\end{array}$ \\
\hline Nivel 2 & $\begin{array}{l}\text { Se espera que el estudiante proponga que las explicaciones se basan en observaciones e inferencias influencia- } \\
\text { das por conocimientos previos, que comprenda que la ciencia argumenta y propone explicaciones e hipótesis } \\
\text { basadas en los experimentos, y que estos son solo modos de obtención de datos «crudos», que se pueden usar } \\
\text { para apoyar (o no) aportes conceptuales, pero que no son capaces de probar algo. }\end{array}$ \\
\hline Nivel 1 & $\begin{array}{l}\text { Se espera que el estudiante proponga explicaciones basadas en la postulación de la existencia de un método } \\
\text { científico único, que adhiera a la idea de que la comprensión científica de la realidad procede directamente } \\
\text { de los datos y resultados obtenidos principal o únicamente por medio de experimentos. En este tipo de } \\
\text { respuestas aparecerá fuertemente la noción de «descubrimiento». }\end{array}$ \\
\hline
\end{tabular}

Cuadro 7.

Categorías elaboradas sobre el aspecto de prácticas y métodos experimentales

\begin{tabular}{|l|l|c|}
\hline \multicolumn{1}{|c|}{ Grupos } & \multicolumn{1}{|c|}{ Categorias creadas } & Nivel \\
\hline $1,2,4,5,6$ y 8 & Aislar y controlar variables & 2 \\
\hline 3 & Observar diferencias estructurales/identificar los sexos & 2 \\
\hline 10 & Simular las condiciones naturales y observar & 1 \\
\hline
\end{tabular}

Cuando se abordó el aspecto empírico del quehacer científico, sus prácticas y sus métodos, las respuestas de los participantes se mostraron más coherentes, como puede observarse tanto en las categorías del cuadro 7 como en sus respuestas textuales. Ejemplo de respuesta considerada como de nivel 2:

Observar si hay diferencias estructurales entre los individuos y después separarlos: (A) individuos con estructuras diferentes, aislar en ambientes separados (impidiendo el apareamiento), (B) dos individuos con estructuras diferentes, aislar en un mismo ambiente (permitiendo el apareamiento) (grupo 5).

A pesar de no tratarse de una argumentación demasiado refinada, esta respuesta se puede encuadrar como de nivel intermedio, pues, en mayor o menor grado, los estudiantes referenciaron factores como la necesidad de aislamiento de los organismos, la realización de más de un experimento (series experimentales), el control de variables y el número de ejemplares.

Ejemplo de respuesta tomada como de nivel 1:

En lugar del frasco con lechuga, se preparaban dos terrarios imitando las condiciones naturales. En uno se coloca un solo individuo y en el otro, dos individuos (grupo 10). 
Estos primeros logros de los profesores en formación constituyen, en nuestra opinión, puntos de anclaje para discutir ideas epistemológicas sobre la práctica científica que incluyan la identificación de problemas, la generación de preguntas de investigación, el análisis y la interpretación de datos, la comunicación y defensa de hipótesis, explicaciones o modelos (Allchin et al., 2014).

En lo que se refiere a este aspecto de NOS, los datos obtenidos permiten evidenciar, de acuerdo con otros trabajos, que los estudiantes tienden a presentar concepciones más adecuadas cuando están frente a un recurso didáctico contextualizado, basado tanto en la historia de la ciencia como en estrategias de indagación (Adúriz-Bravo, 2011, 2015b; Kendig, 2013) 2011.

El último aspecto NOS que discutimos con los profesores participantes versó sobre el papel de la comunicación y la persuasión en el desarrollo del conocimiento científico. Para ello se elaboró la pregunta expuesta en el cuadro 8 .

\section{Cuadro 8 .}

Rúbrica sobre la persuasión y la comunicación científica

\begin{tabular}{|c|c|}
\hline \multicolumn{2}{|c|}{$\begin{array}{l}\text { Bonnet logró lo que otros naturalistas que lo antecedieron no habian logrado. A través de un experimento pudo observar el na- } \\
\text { cimiento de un áfido a partir de otros que no se habian apareado. Frente a este evento empiricamente evidenciado, Bonnet se } \\
\text { pregunta: ¿qué hacer ahora? (¿lo publico?, ¿lo discuto con la comunidad de estudiosos?, ¿elaboro una replicación?, etc.) y ¿por qué } \\
\text { preferir un camino u otro? }\end{array}$} \\
\hline Nivel 3 & $\begin{array}{l}\text { Se espera que el estudiante argumente sobre la necesidad de revisión y repetición de observaciones y } \\
\text { experimentos y sobre el papel de la divulgación de los datos, métodos y procedimientos dentro de la } \\
\text { comunidad académica (comunicación entre pares). El estudiante resalta el papel de la argumentación y la } \\
\text { retórica en la persuasión de los colegas. }\end{array}$ \\
\hline Nivel 2 & $\begin{array}{l}\text { Se espera que el estudiante reconozca la necesidad de la revisión por pares, el papel de la comunicación } \\
\text { académica y la validación de métodos y procedimientos empíricos. }\end{array}$ \\
\hline Nivel 1 & $\begin{array}{l}\text { Los aspectos de comunicación y persuasión no aparecen o están muy desdibujados, poniéndose el énfasis } \\
\text { en el descubrimiento y la demostración. }\end{array}$ \\
\hline
\end{tabular}

Cuadro 9.

Categorías elaboradas sobre el aspecto de persuasión y comunicación científica

\begin{tabular}{|l|l|c|}
\hline \multicolumn{1}{|c|}{ Grupos } & \multicolumn{1}{|c|}{ Categorías creadas } & Nivel \\
\hline 2 & Publicar los datos obtenidos & 2 \\
\hline 5 y 9 & Discutir con la comunidad científica & 2 \\
\hline $1,3,4,6,7,8$ y 10 & Realizar una replicación/repetición & 1 \\
\hline
\end{tabular}

La concepción de los participantes con relación a este aspecto de NOS se reveló parcialmente adecuada solo en tres grupos (cuadro 9), lo que da indicio de que este aspecto epistemológico es de los más complejos de incorporar. Las respuestas presentadas a continuación ejemplifican la posición de los profesores en este aspecto.

Ejemplo de respuesta entendida como de nivel 2:

Repetir el experimento exactamente igual que el experimento anterior y luego publicarlo para discutirlo con la comunidad científica (grupo 2).

Ejemplo de respuesta clasificada como de nivel 1:

Lo replicaría para poder determinar si la experiencia es válida o fue casualidad (grupo 1). 
La mayoría de los participantes consideró la relevancia de la replicación y la repetición experimental para obtener datos más consistentes con el problema investigado, pero solo unos pocos mencionaron también la importancia de comunicar los resultados obtenidos y discutirlos con la comunidad de científicos. Al trabajar con el OVA, los profesores en formación se mostraron conscientes del papel de las prácticas y los métodos empíricos, pero advirtieron poco la necesidad de comunicación de los resultados observados a pares «validadores».

Este último conjunto de ideas NOS es particularmente importante porque la comunicación (publicación de artículos, intercambio de correspondencia, comunicación institucional, congresos científicos, etc.) es una práctica fundamental para el desarrollo científico (Krogh y Nielsen, 2013), en cuanto que permite tanto la organización sistemática de las evidencias como el convencimiento de los colegas (búsqueda de «adeptos»). En todo ello, la revisión por pares y la apertura a la crítica colectiva actúan como sistema de control que permite identificar y aislar errores, detectar preconcepciones y sesgos y proporcionar conclusiones más fundamentadas (Allchin, 2013).

\section{Percepciones sobre la aplicabilidad del OVA}

Por tratarse de una propuesta innovadora de enseñanza de la naturaleza de la ciencia, evaluamos también la percepción de los profesores participantes que interactuaron con el OVA en relación con su usabilidad en la práctica docente. Además, se indagó acerca de las posibilidades de aplicación del recurso en el contexto de la educación secundaria. Para ello se elaboró un instrumento de recolección de datos con cinco preguntas abiertas y se lo administró individualmente a los participantes después de la práctica con el OVA. En las figuras 3 a 7 se consignan las cinco preguntas y las frecuencias de las respuestas.

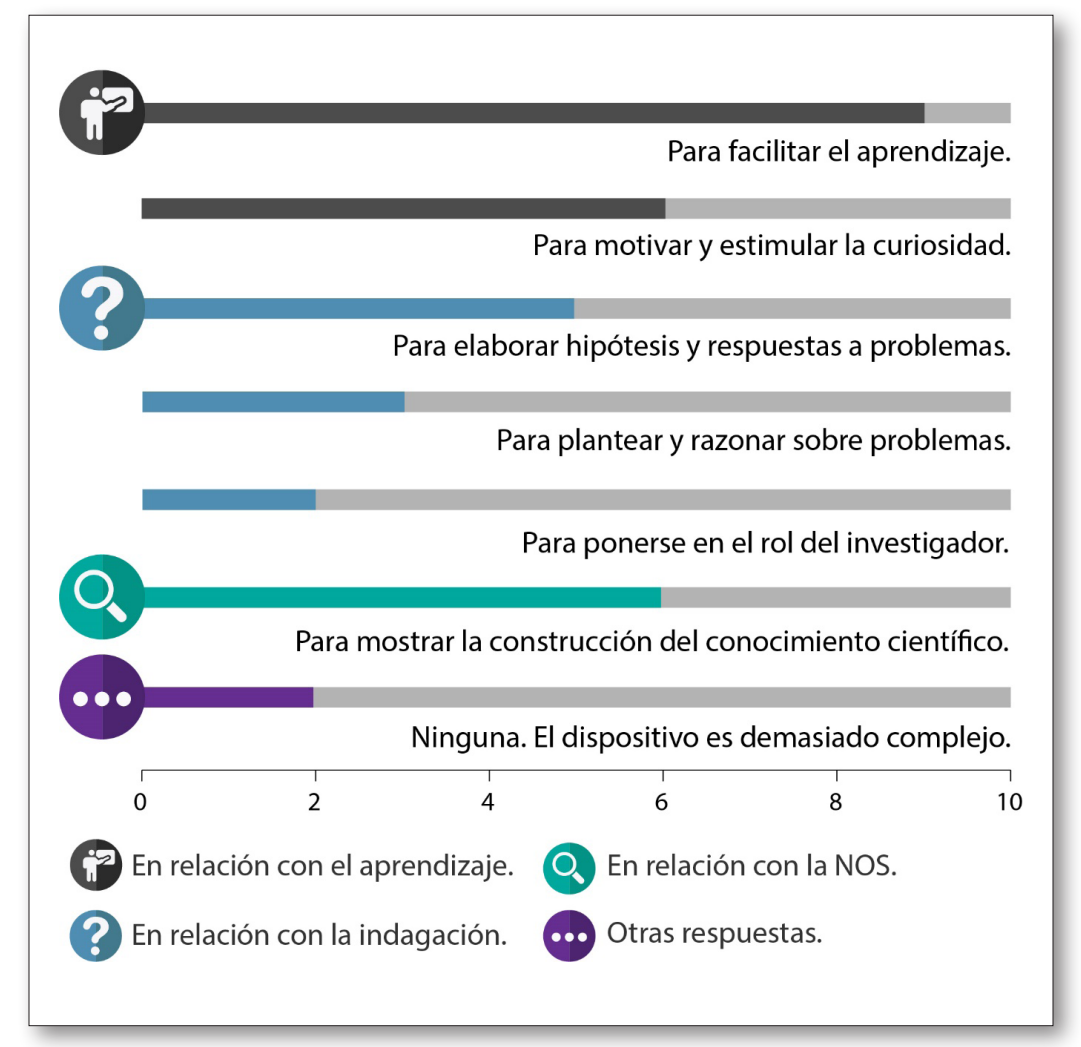

Fig. 3. Pregunta 1: ¿Qué posibilidades identificas en la aplicación de esta metodología en las clases de biología de escuela media? 


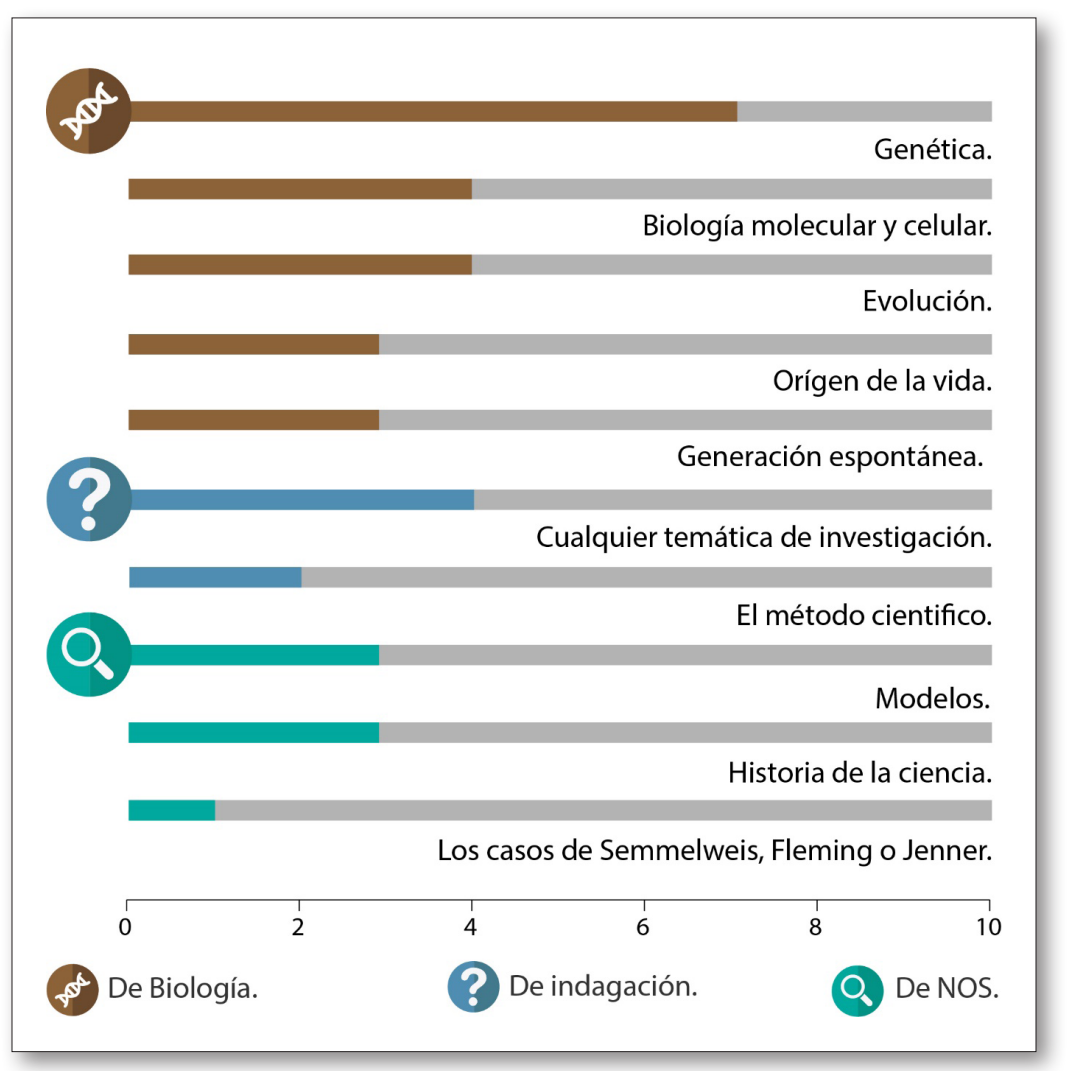

Fig. 4. Pregunta 2: ¿Qué otras temáticas podrías trabajar con este recurso?

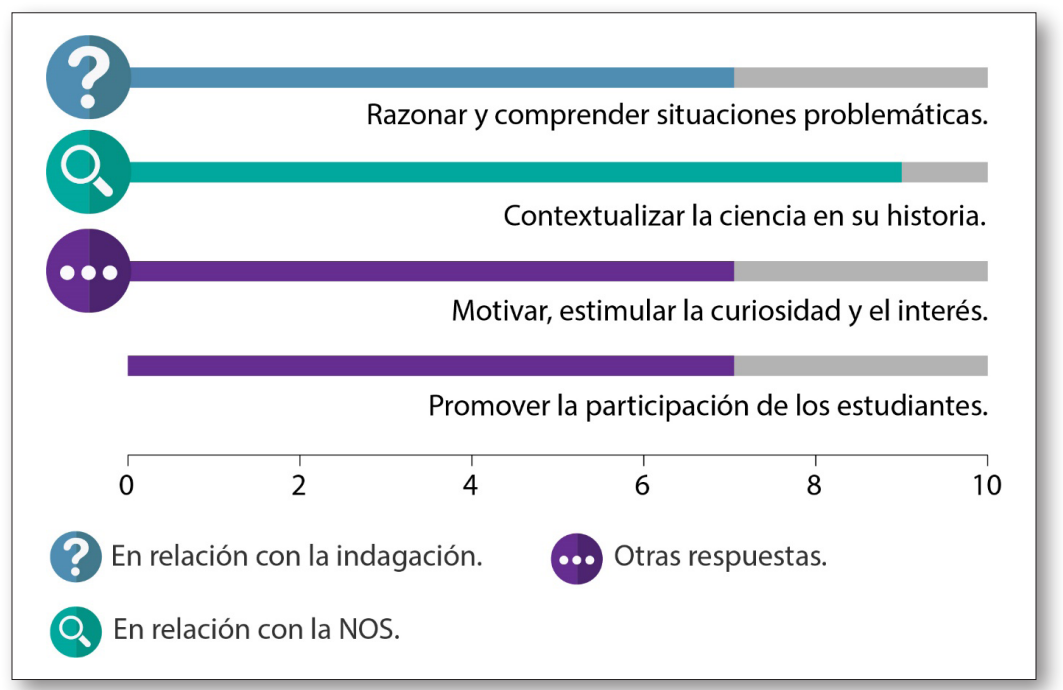

Fig. 5. Pregunta 3: ¿Qué beneficios crees que aporta este recurso para el aprendizaje? 


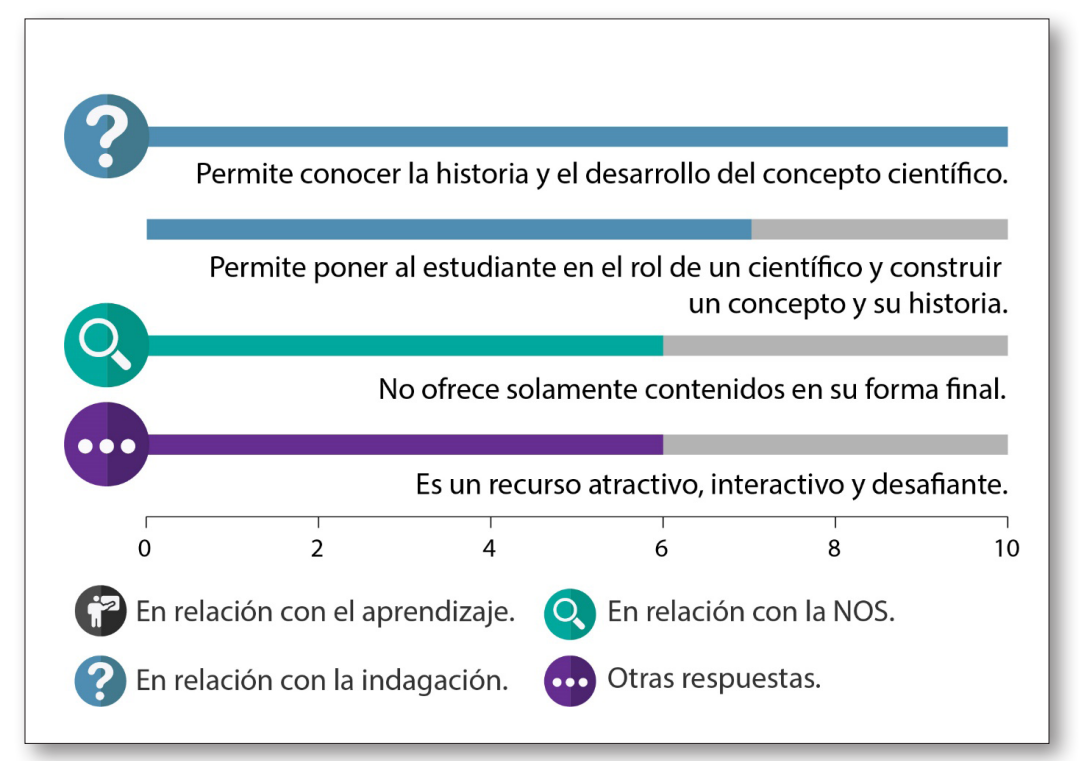

Fig. 6. Pregunta 4: ¿Qué diferencias ves en la forma en que esta narrativa aborda el concepto de reproducción asexual en comparación con los libros de texto?

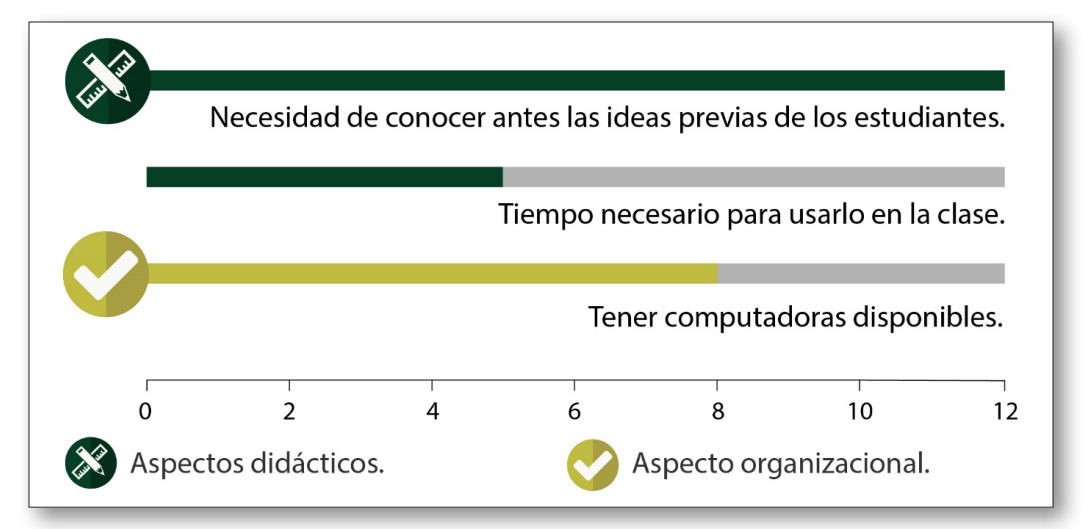

Fig. 7. Pregunta 5: ¿Cuáles serían los aspectos negativos del uso del recurso?

Con base en la categorización de las respuestas, se puede colegir que en general el OVA tuvo aceptación positiva por parte de estos futuros profesores. Es interesante notar también que, en muchos casos, se establece una relación sustantiva entre las posibilidades y beneficios del recurso virtual y las cuestiones abordadas de naturaleza de la ciencia.

En lo que se refiere a las posibilidades de aplicación en la enseñanza de la biología en la escuela secundaria (pregunta 1) y a los beneficios que el dispositivo podría aportar al aprendizaje (pregunta 3), las respuestas son diversas, pero con elementos en común, como el énfasis en la indagación basada en problemas o el reconocimiento de que el recurso permite tratar la historicidad de la ciencia.

La mayoría de los profesores en formación expuestos al recurso virtual reconocen que tiene potencial para trabajar aspectos clave del quehacer científico (elaboración de hipótesis, construcción de conceptos, ubicar a los estudiantes en el lugar del científico). Es posible inferir que tales impresiones se apoyan en el carácter contextual del OVA, dado que nuestra propuesta pretendía explícitamente crear un ambiente de integración entre la historia de la ciencia y los elementos de la indagación científica en el entorno virtual. En este sentido, los resultados se condicen con la literatura que señala que la contextualización histórica, cuando se encuentra vinculada a aspectos de indagación, ayuda al aprendizaje 
de aspectos del quehacer científico (Berçot et al., 2018; García-Martínez e Izquierdo-Aymerich, 2014; Lozano et al., 2016).

También se mencionan ventajas educativas generales del OVA, como la motivación y la contribución al aprendizaje; en efecto, es bien conocido el carácter motivador de los recursos virtuales de aprendizaje (Beluce y Oliveira, 2015), así como la posibilidad de estas herramientas de promover procesos de aprendizaje (Berçot et al., 2013).

Cuando se indagó a los participantes acerca de la posible aplicación del OVA a otras temáticas del currículo de biología (pregunta 2), la mayoría de los participantes se refirió a contenidos centrales de la disciplina. Sin embargo, algunas respuestas se centraron en elementos de la historia de la ciencia, como el episodio de Ignác Semmelweis y la fiebre puerperal, estudio ya desarrollado con fines educativos (Revel Chion et al., 2013), enfoques sobre el uso de los modelos en la ciencia (Adúriz-Bravo, 2015a) y temáticas de indagación vinculadas con la idea, aún muy vigente entre el profesorado, del «método científico".

Se indagó asimismo sobre aquellos elementos del OVA que trascienden los libros de texto utilizados en la enseñanza de la biología (pregunta 4). Las respuestas se alinean con lo que se vio en las cuestiones 1 y 3: el OVA se distingue por no presentar los contenidos descontextualizados ni en su «forma final».

En un análisis general de las respuestas podemos concluir que la gran mayoría de los profesores participantes establece vínculo entre la contextualización histórica propuesta y la posibilidad de aprendizaje de la construcción conceptual. Las respuestas textuales de dos de los participantes nos permiten ejemplifican ese punto:

Esta metodología no se centra en dar conceptos como en los libros, que a veces pueden llegar a aparecer como si fueran un diccionario o enciclopedia. La narrativa permite reconocer los fenómenos [...] que requieren esfuerzo (estudiante 18).

Permite entender cómo se llega al concepto y no solo a su definición [...]. De esta manera, se obtiene una mayor comprensión del tema y es más creíble que si solo te explican el fenómeno (estudiante 22).

El último punto del cuestionario buscó identificar alguna posible dificultad de implementación del OVA en el aula. Reconocer aspectos negativos que los futuros profesores prevén es relevante para reformular y proponer mejoras en él. La principal crítica tiene que ver con el tiempo como limitante para la implementación de una propuesta de indagación con historia de la ciencia, lo que es entendible desde la perspectiva de que los currículos vigentes tienen una alta densidad de contenido, pero también desde la concepción, extendida entre el profesorado, de que lo importante es el aprendizaje conceptual de la biología en sentido estricto.

En relación con esta preocupación por la gestión del tiempo de clase, en la puesta en común final con los participantes se discutió que, si bien ellos trabajaron dos horas completas con el OVA, este se puede trabajar asincrónicamente, fraccionado en tareas. Se intentó enfatizar que la forma en que utilicemos el recurso dependerá de los criterios impuestos por el docente del curso. Nuestra propuesta no pretende constituirse en un "protocolo didáctico» rígido, sino pasar a ser una herramienta virtual disponible para la contextualización de la biología escolar en episodios de la historia de la ciencia y con el propósito declarado de discutir aspectos de NOS.

\section{CONSIDERACIONES FINALES}

La literatura sobre filosofía e historia de la ciencia orientadas hacia la enseñanza de las ciencias está bien provista de ejemplos exitosos y de evidencias favorables en torno al aprendizaje integrado de y sobre la ciencia. Sin embargo, el elemento diferencial de nuestra propuesta reside en integrar episodios de la historia de la ciencia, elementos de indagación científica y aspectos de la naturaleza de la ciencia 
en un objeto virtual de aprendizaje. Además, al producir un recurso virtual libre y de acceso gratuito, pretendemos contribuir a esta área de trabajo introduciendo las TIC, lo que es una demanda actual.

La aplicación del OVA con profesores en formación nos permitió entrever que el recurso cumple su propósito, pues hizo posible caracterizar y discutir las concepciones de los participantes sobre la naturaleza del quehacer científico por medio de cuestiones abiertas con carácter de indagación. Los participantes presentaron concepciones de carácter «intermedio» en la mayoría de los aspectos NOS explorados, lo que constituye un muy buen punto de partida para introducir ideas epistemológicas «clave» en su formación.

Sobre una ulterior aplicación del OVA a la educación secundaria, la gran mayoría de los participantes reconocieron sus potencialidades como recurso virtual. Se abre así la puerta a la investigación crítica de futuras implementaciones en este nivel de la mano de profesores expertos y novatos.

No obstante lo señalado, somos conscientes de las limitaciones de este estudio, toda vez que nuestro ensayo del OVA se ha restringido a una sola clase de nivel superior no universitario. Sin perjuicio de este reconocimiento, estamos persuadidos del potencial del OVA como herramienta alternativa para la enseñanza de aspectos de la naturaleza de la ciencia. Esta afirmación se sustenta en el hecho de que, a pesar de todos los avances alcanzados en las últimas décadas, la formación inicial docente aún adolece de la adecuada atención al aprendizaje de NOS. Muchos profesores llegan a las aulas con una base metacientifica poco satisfactoria para enfrentar la necesaria reflexión acerca del quehacer científico (prescrita en el currículo de ciencias vigente en Argentina). A causa de ello, sobreestiman en el aula las prácticas experimentales y entronizan un supuesto «método científico», perpetuando así una visión empírico-inductivista de la ciencia que es incompatible con una alfabetización científica de calidad.

En función de las consideraciones anteriores, nuestra propuesta aporta un material y una modalidad alternativas para la formación inicial docente en NOS, con el objetivo de paliar en parte las recurrentes referencias a visiones deformadas acerca de la ciencia.

\section{REFERENCIAS}

Abell, S. y Smith, D. (1994). What is science?: Preservice elementary teachers' conceptions of the nature of science. International Journal of Science Education, 16, 475-487.

https://doi.org/10.1080/0950069940160407

Acevedo-Díaz, J. A., García-Carmona, A. y Aragón, M. M. (2017). Historia de la ciencia para enseñar naturaleza de la ciencia: Una estrategia para la formación inicial del profesorado de ciencia. Educación Química, 28, 140-146. http://dx.doi.org/10.1016/j.eq.2016.12.003

Adúriz-Bravo, A. (2009). La naturaleza de la ciencia «ambientada» en la historia de la ciencia. Enseñanza de las Ciencias, extra VIII Congreso, 1177-1180.

Adúriz-Bravo, A. (2011). Use of history of science in the design of research-informed NOS materials for teacher education. En P. Kokkotas, K. Malamitsa y A. Rizaki (Eds.), Adapting Historical Knowledge Production to the Classroom (pp. 195-204). Rotterdam: Sense Publishers.

Adúriz-Bravo, A. (2015a). Pensamento «basado en modelos» en la enseńanza de las ciencias naturales. Revista del Instituto de Investigaciones en Educación, 6, 20-31.

http://dx.doi.org/10.30972/riie.063680

Adúriz-Bravo, A. (2015b). Teaching the nature of science with scientific narratives. Interchange, 45, 167-184. https://doi.org/10.1007/s10780-015-9229-7

Allchin, D. (2013). Teaching the nature of science: Perspectives and resources. Saint Paul: SHiPS Education Press. 
Allchin, D., Andersen, H. y Nielsen, K. (2014). Complementary approaches to teaching nature of science: Integrating student inquiry, historical cases and contemporary cases in classroom practice. Science Education, 98, 461-486. https://doi.org/10.1002/sce.21111

Allen, D. y Tanner, K. (2006). Rubrics: Tools for making learning goals and evaluation criteria explicit for both teachers and learners. CBE Life Sciences Education, 5, 197-203. https://doi.org/10.1187/cbe.06-06-0168

Andrade, H., Du, Y. y Wang, X. (2008). Putting rubrics to the test: The effect of a model, criteria generation and rubric-referenced self-assessment on elementary school students' writing. Educational Measurement: Issues and Practice, 27, 3-13.

https://doi.org/10.1111/j.1745-3992.2008.00118.x

Bardin, L. (1977). L'Analyse de Contenu. París: PUF.

Beluce, A. y Oliveira, K. de (2015). Students' motivation for learning in virtual learning environments. Paidéia, 25, 105-113. https://doi.org/10.1590/1982-43272560201513

Berçot, F. F., Cortez, E. y Prestes, M. E. B. (2018). Abraham Trembley (1710-1784) and the creature that defies classification: Nature of science and inquiry through a historical narrative. En M. E. B. Prestes y C. C. Silva (Eds.), Teaching science with context (pp. 161-190). Dordrecht: Springer. https://doi.org/10.1007/978-3-319-74036-2_11

Berçot, F. F., Fidalgo-Neto, A., Lopes, R., Faggioni, T. y Alves, L. (2013). Virtual immunology: Software for teaching basic immunology. Biochemistry and Molecular Biology Education, 41, 377-383. https://doi.org/10.1002/bmb.20733

Berçot, F. F. y Prestes, M. E. B. (2017). O enigma do pulgão. En B. A. Moura y T. C. M. Forato (Eds.), Histórias das ciências, epistemologia, gênero e arte: Ensaios para a formação de professores (pp. 11-35). Santo André: Editora da UFABC.

Bonnet, C. (1745). Traité d'insectologie ou Observations sur les pucerons. París: Chez Durand.

Erduran, S. y Wong, S. (2013). Science curriculum reform on «Scientific Literacy for All» across national contexts: Case studies of curricula from England, Wales and Hong Kong. En N. Mansour y R. Wegerif (Eds.), Science education for diversity: Theory and practice (pp. 179-201). Dordrecht: Springer. https://doi.org/10.1007/978-94-007-4563-6_9

Fernández, I., Gil Pérez, D., Carrascosa, J., Cachapuz, A. y Praia, J. (2002). Visiones deformadas de la ciencia transmitidas por la enseñanza. Enseñanza de las Ciencias, 20, 477-488. https://doi.org/10.13140/2.1.1069.5367

Fouad, K., Masters, H. y Akerson, V. (2015). Using history of science to teach nature of science to elementary students. Science \& Education, 24, 1103-1140. https://doi.org/10.1007/s11191-015-9783-5

García-Martínez, Á. y Izquierdo Aymerich, M. (2014). Contribución de la historia de las ciencias al desarrollo profesional de docentes universitarios. Enseñanza de las Ciencias, 32, 265-281. https://doi.org/10.5565/rev/ensciencias. 758

Hafner, J. C. y Hafner, P. M. (2003). Quantitative analysis of the rubric as an assessment tool: An empirical study of student peer-group rating. International Journal of Science Education, 25, 1509-1528. https://doi.org/10.1080/0950069022000038268

Kampourakis, K. (2016). The "general aspects» conceptualization as a pragmatic and effective means to introducing students to nature of science. Journal of Research in Science Teaching, 53, 667-682. https://doi.org/10.1002/tea.21305 
Kendig, C. (2013). Integrating history and philosophy of the life sciences in practice to enhance science education: Swammerdam's Historia Insectorum Generalis and the case of the water flea. Science \& Education, 22, 1939-1961. https://doi.org/10.1007/s11191-013-9596-3

Krogh, L. y Nielsen, K. (2013). Introduction: How science works — and how to teach it. Science \& Education, 22, 2055-2065. https://doi.org/10.1007/s11191-013-9582-9

Lozano, E., Bahamonde, N. y Adúriz-Bravo, A. (2016). Análisis histórico-epistemológico sobre los modelos de membrana celular para enseñar biología celular y naturaleza de la ciencia al profesorado. Filosofia e História da Biologia, 11(1), 49-68.

Matthews, M. (2012). Changing the focus: From nature of science (NOS) to features of science (FOS). En M. S. Khine (Ed.), Advances in nature of science research: Concepts and methodologies (pp. 3-26). Dordrecht: Springer. https://doi.org/10.1007/978-94-007-2457-0_1

Moskal, B. y Leydens, J. (2000). Scoring rubric development: Validity and reliability. Practical Assessment, Research \& Evaluation, 7, 1-10. https://doi.org/10.1016/j.asw.2010.01.003

Mulvey, B. y Bell, R. (2016). Making learning last: Teachers' long-term retention of improved nature of science conceptions and instructional rationales. International Journal of Science Education, 39, 62-85. https://doi.org/10.1080/09500693.2016.1267879

Norris, S., Guilbert, S., Smith, M., Hakimelahi, S. y Phillips, L. (2005). A theoretical framework for narrative explanation in science. Science Education, 89, 535-563. https://doi.org/10.1002/sce.20063

Prestes, M. E. B. (2003). A biologia experimental de Lazzaro Spallanzani (1729-1799) (tesis doctoral). Universidade de São Paulo, Faculdade de Educação.

Prestes, M. E. B. (2008). Definição e classificação de insetos por René-Antoine Ferchault de Réaumur. Filosofia e História da Biologia, 3(1), 263-284.

Réaumur, R.-A. F. de (1737-1742). Memoires pour servir a l'histoire des insectes. Tomos 3 y 6. París: L'Imprimerie Royale.

Reddy, Y. y Andrade, H. (2010). A review of rubric use in higher education. Assessment and Evaluation in Higher Education, 35, 435-448. https://doi.org/10.1080/02602930902862859

Revel Chion, A., Meinardi, E. y Adúriz-Bravo, A. (2013). Elementos para un análisis histórico-epistemológico del concepto de salud con implicaciones para la enseñanza de la biología. Filosofia e História da Biologia, 8(1), 1-19.

Roach, L. y Wandersee, J. (1995). Putting people back into science: Using historical vignettes. School Science and Mathematics, 95, 365-370. https://doi.org/10.1111/j.1949-8594.1995.tb15802.x

Rudge, D., Cassidy, D., Fulford, J. y Howe, E. (2012). Changes observed in views of nature of science during a historically based unit. Science \& Education, 23, 1879-1909. https://doi.org/10.1007/s11191-012-9572-3

Sigrist, R. (1994). Science et société à Genàve au Xviır siècle: L'exemple de Charles Bonnet. En M. Buscaglia et al. (Eds.), Charles Bonnet, savant et philosophe (1720-1793) (pp. 19-39). Ginebra.

Sigrist, R. (2001). L'expérimentation comme rhétorique de la preuve: L'exemple du Traité d'Insectologie de Charles Bonnet. Revue d'Histoire des Sciences, 54, 419-449.

https://doi.org/10.3406/rhs.2001.2133 
Terrall, M. (2010). Following insects around: Tools and techniques of eighteenth-century natural history. British Journal for the History of Science, 43, 573-588.

https://doi.org/10.1017/S0007087410001287

Wiley, D. (2002). Connecting learning objects to instructional design theory: A definition, a metaphor and a taxonomy. En D. Wiley (Ed.), The instructional use of learning objects. Bloomington: Agency for Instructional Technology. 


\section{Nature of science in a virtual learning object for prospective science teachers}

\author{
Filipe Faria Berçot \\ Instituto de Biociencias, \\ Universidad de San Pablo, \\ San Pablo, Brasil. \\ bercot@ib.usp.br
}

\author{
Andrea Revel Chion \\ Instituto CeFIEC, \\ Universidad de Buenos Aires, \\ Buenos Aires, Argentina. \\ andrearevelchion@gmail.com
}

\author{
Agustín Adúriz-Bravo \\ CONICET/Instituto CeFIEC, \\ Universidad de Buenos Aires, \\ Buenos Aires. Argentina. \\ aadurizbravo@cefiec.fcen.uba.ar
}

The aim of this piece of research was to validate a virtual learning object (VLO) constructed on the basis of an episode of the history of biology in Europe in the eighteenth century and directed to teaching some key ideas of the nature of science (NOS). A first implementation of this VLO in prospective science teacher education permitted the evaluation of its pros and cons in a realistic setting.

The abundant literature on the uses of the history and philosophy of science in science education reports many successful innovations oriented towards teaching science and NOS in an integrated way. The differential element in our proposal resides in integrating episodes from the history of science, elements of scientific inquiry, and aspects of the nature of science using ICTs.

The historical episode that we chose for our VLO concerns the studies of Genevan naturalist Charles Bonnet (1720-1793), who provided sound arguments in favour of the idea of asexual reproduction in animals. From this episode, we developed the VLO structuring it as an interactive website. We followed some of the theoretical and methodological guidelines that are available in the literature on the use of narratives in science education, and we elaborated a "science story» called The enigma of plant-lice. The story was composed of short texts written in informal language and including historical materials from primary sources. Our science story was interspersed with seven tasks for inquiry, based on «authentic» historical problems, and directed to the discussion of some central epistemic aspects of NOS.

The web-based science story with the corresponding inquiry tasks was piloted in an undergraduate course on didactics of biology forming part of a programme of pre-service science teacher education in Buenos Aires, Argentina. Twenty-nine student teachers (average age 21) participated in a 2-hour lesson on the use of the history of science in science teaching, delivered by the first author of this article. The participants were organised in 10 groups of 3 students each. Groups were required to sequentially read and discuss fragments of the science story and, between two successive fragments, to answer seven questions for inquiry, which were labelled as «Moments to think». The 29 prospective teachers were also asked to individually answer a survey of 7 questions asking for their views on the applicability and educational quality of the VLO. Group answers to the inquiry questions and individual answers to the survey constituted the corpus for our empirical study, which followed the principles of content analysis.

The results that we report here show that our VLO satisfactorily serves its purpose: its implementation in the lesson allowed student teachers to formulate and discuss their views on the nature of science-in-the-making around aspects such as theory-ladenness of observation, methodologies, and communication and persuasion. More than half of the participants showed «intermediate» NOS conceptions, which is itself a solid starting point for the introduction of NOS «key ideas» in their professional preparation.

Participating student teachers also recognised the potential value of our VLO for secondary education, identifying the curriculum topics where it could be used, the inquiry competencies that it could foster, and the NOS aspects for which it could be most suitable.

Notwithstanding the limitations of our study, with preliminary results that cannot be generalised, we are persuaded of the pertinence of the VLO as a tool to teach NOS. In spite of all the reported achievements in NOS, pre-service science teacher education still needs to pay more attention to reflection on science with the aid of the philosophy of science. Many student teachers come to the courses with naif or distorted ideas on the functioning of science as a sophisticated endeavour: they overestimate the value of experimental practices and stick to the positivistic construct of «scientific method». Our proposal makes an online resource that tackles with such ideas available. 